\title{
A PRODUÇÃo CIENTÍFICA SOBRE A APLICAÇÃo DA HIPÓTESE DE PORTER ENTRE OS ANOS DE 2000 A 2015
}

\section{THE SCIENTIFIC PRODUCTION ON THE APPLICATION OF PORTER'S HYPOTHESIS BETWEEN THE YEARS 2000 TO 2015}

\author{
Hillary Lapas Fujihara \\ Discente do Mestrado Profissional em Administração da Unioeste. \\ E-mail: hillary_lapas@hotmail.com \\ Elizandra da Silva \\ Docente do Mestrado Profissional em Administração da Unioeste. Doutora em \\ Administração. E-mail: elizandra.silva@unioeste.br
}

Geysler Rogis Flor Bertolini

Docente do Mestrado Profissional em Administração e do Mestrado em Contabilidade da Unioeste. Doutor em Engenharia de Produção. E-mail: geysler_rogis@yahoo.com.br

Recebido em: 20/04/2017

Aprovado em: 02/08/2017

Doi: $10.5585 /$ rdb.v18i7.661

RESUMO: A presente pesquisa objetiva analisar a aplicação da Hipótese de Porter e sua relevância na inovação empresarial em decorrência das legislações ambientais evidenciados nos artigos disponíveis na Base Ebsco entre os anos de 2000 a 2015. A pesquisa caracteriza-se como levantamento bibliográfico exploratório, por proporcionar aos pesquisadores maior contato com o assunto pesquisado. Para tanto foi desenvolvido uma pesquisa na Ebsco de agosto a novembro de 2015 e posteriormente foram analisados 82 artigos de diferentes Instituições de Ensino e países. Verificou-se que apesar de ser muito criticada a hipótese consegue atingir seu objetivo nos mais diversos setores, entretanto a maioria das pesquisas concentra-se no setor industrial.

Palavras-chave: Hipótese de Porter. Sustentabilidade. Inovação. Regulamentação Ambiental.

\begin{abstract}
This research aims to analyze the application of Porter's Hypothesis and its relevance in business innovation due to environmental laws evidenced in articles available in Ebsco Basis between the years 2000 to 2015. The research is characterized as exploratory bibliographical, by providing researchers with greater contact with the subject researched. For this purpose was developed a search on Ebsco from August to November of 2015 and later analyzed 82 articles from different institutions and countries. It was found that despite being highly criticized the hypothesis can reach your goal in the most diverse sectors, however most research focuses on the industrial sector.
\end{abstract}

Keywords: Porter Hypothesis; Sustainability; Innovation; Environmental Regulation 
SUMÁRIO: Introdução; 1. Referencial teórico; 2. Métodos; 3. Resultados; 3.1. Análise quantitativa dos artigos; 3.2. Análise qualitativa dos artigos; Conclusão; Referências bibliográficas.

\section{INTRODUÇÃO}

Aquino, Cavalheiro e Pellenz (2016) afirmam que pela primeira vez em todo o período histórico há uma grande preocupação com a natureza e com a forma que o homem relaciona-se com ela. Gerando uma consciência nos diversos setores, saberes e autoridades científicas.

Para Kozluk e Zipperer (2013) a abordagem tradicional vê as políticas ambientais como um fardo sobre a economia empresarial, principalmente no curto e médio prazo, à medida que aumentam os custos de produção. Seguindo essa linha de pensamento alguns empresários acreditam que a regulamentação ambiental afeta negativamente a competitividade, pois ele terá que fazer investimentos em alguns pontos da empresa para atender a regulamentação e alguns que ele considera prioridade acabarão ficando para depois, porém a Hipótese de Porter contesta este ponto de vista, propondo uma perspectiva dinâmica na qual inovações induzidas por políticas ambientais podem compensar os custos provenientes da regulação (PORTER; VAN DER LINDE, 1995).

A Hipótese de Porter (HP) foi proposta originalmente por Porter (1991) e Porter e van der Linde (1995), nesta hipótese os autores argumentam que quando a regulamentação ambiental é bem trabalhada beneficiará tanto o meio ambiente, quanto a empresa. Pois, para Ansanelli (2011), Ashford (2000) e Porter e van der Linde (1995), a regulamentação ambiental deve estimular às preocupações com o meio ambiente e às mudanças tecnológicas, influenciando diretamente na quantidade produzida e qualidade, consequentemente na competitividade.

Caliendo e Muniz (2015), afirmam que a há uma inter-relação entre política fiscal e desenvolvimento tecnológico empresarial, pois a política deve eliminar barreiras ao investimento e competitividade empresarial. Porter e van der Linde (1995) argumentam que as normas ambientais quando adequadamente projetadas podem desencadear inovação que pode parcialmente ou total mais do que compensar os custos do cumprimento com eles. Essas "compensações de inovação", não reduzem o custo líquido de cumprir as leis ambientais, mas conduzem às vantagens absolutas sobre as empresas em países estrangeiros não sujeitos a semelhantes regulamentos.

Entretanto, está hipótese ainda não é muito pesquisada no Brasil, fator este evidenciado por esta pesquisa, uma vez que inicialmente pesquisou-se 121 periódicos brasileiros referentes à área de Administração, Ciências Contábeis, Ciências Econômicas e Turismo, classificadas entre A2 e B3 pela Capes. Utilizando a combinação "Hipótese de Porter" obteve-se 33 artigos e "Porter Hypothesis" 13, no entanto todos foram rejeitados, pois nenhum tratava sobre a Hipótese de Porter.

Após ter sido necessário rejeitar todos os 46 artigos encontrados, optou-se por realizar a pesquisa em uma base internacional, então escolheu-se a base de pesquisa Ebsco. Utilizando as mesmas combinações de palavras, encontrou-se 83 artigos de 56 revistas e 24 países distintos, mas, foi necessário rejeitar o único artigo encontrado utilização a combinação de palavras em português, e assim foram analisados 82 artigos de 55 revistas e 23 países diferentes. Objetivando analisar a aplicação da Hipótese de Porter e sua relevância na inovação empresarial em decorrência das legislações ambientais nos artigos disponíveis na Base Ebsco entre os anos de 2000 a 2015.

O presente estudo caracterização como levantamento bibliográfico exploratório, pois pretendeu-se ter maior proximidade com o assunto, por meio da análise dos artigos disponíveis na base Ebsco. 
O mesmo estrutura-se em quatro seções, além da introdução, na próxima seção, apresenta-se o referencial teórico baseado na Hipótese de Porter; na terceira seção, descrevem-se os procedimentos metodológicos adotados para o desenvolvimento deste artigo; na quarta apresentam-se os resultados obtidos por meio de análise dos 82 artigos utilizados como base para o desenvolvimento desta pesquisa; e, na quinta, tem-se as considerações finais, abrangendo conclusão e sugestões para futuras pesquisas.

\section{REFERENCIAL TEÓRICO}

Segundo Ambec, Cohen, Elgie e Lanoie (2013) as empresas enfrentam imperfeições do mercado, como a assimetria de informações, inércia organizacional e controle de problemas. Dessa forma, a regulamentação ambiental pressionará as empresas a superar algumas deficiências do mercado e prosseguir de outra forma garantindo a criação de oportunidades.

Porter (1991) e Porter e van der Linde (1995) consideram que não investir em inovações gera um custo, que os autores denominam como custo de conformidade e este custo é superior ao custo de investimento em inovações ambientais, pois estes investimentos promoverão a inovação, que por sua vez aumentará a eficiência dos recursos e valor do produto, compensando os custos e melhorando a produtividade da empresa.

Maçaneiro, Cunha, Kuhl e Cunha (2015, p. 70), complementam afirmando que "uma regulamentação ambiental mais rigorosa e específica pode forçar as empresas poluentes a buscarem inovações para reduzir o custo da melhoria do impacto ambiental, incrementar também a competitividade e levar a uma relação positiva entre o desempenho ambiental e econômico".

Para Porter e van der Linde (1995) há duas formas de inovação em decorrência da regulamentação ambiental, a primeira prepara a empresa sobre como lidar com problemas ambientais, como, poluição, pois aumenta o conhecimento dela sobre o assunto e a segunda faz com que a empresa minimize os impactos ambientais causados pelo seu produto, melhorando, assim a qualidade do produto oferecido pela empresa.

Devido a globalização, aumento da competitividade, aumento da cobrança dos consumidores e aumento da preocupação com questões ambientais, tornou-se imprescindível analisar o desempenho das empresas e elaborar políticas, assim, a HP tem sido o foco de muitas contribuições empíricas desde a sua origem. (RUBASHKINA; GALEOTTI; VERDOLINI, 2015).

Jaffe e Palmer (1997) desenvolveram três distintas demonstrações sobre a eficiência da HP (narrow, weak e strong). A primeira é denominada de "narrow" HP, nesta demonstração os autores afirmam que uma regulamentação ambiental flexível aumenta os incentivos à inovação empresarial. Na segunda, a "weak" HP, consideram o efeito positivo da regulamentação ambiental sobre inovação ambiental, mesmo quando tais inovações vem a um custo de oportunidade que excede os seus benefícios para a empresa. Por fim, a "strong" HP, postulam que a inovação induzida pela regulamentação ambiental poderia compensar custos regulamentares adicionais e, consequentemente, aumentar a competitividade e produtividade organizacional.

Broberg, Marklund, Samakovlis e Hammar (2013) afirmam que a HP frequentemente é considerada como "ganha-ganha", uma vez que sugere que as empresas sujeitas a uma regulamentação mais rígida pode realmente se beneficiar, por meio de uma maior competitividade, ao mesmo tempo que melhora o ambiente.

Porter e Van der Linde (1995) argumentam que a regulamentação ambiental deveria incentivar os investimentos em mudanças de produtos e processos (prevenção da poluição), para ter um melhor aproveitamento dos recursos, em vez de usar estes investimentos para tratamentos secundários (controle da poluição), que é mais dispendiosa. 


\section{MÉTODOS}

A presente pesquisa caracteriza-se como exploratório, pois de acordo com Collis e Hussey (2005), este tipo de pesquisa tem por objetivo proporcionar aos autores maior familiaridade com o assunto estudado.

De acordo com Gil (2008), a pesquisa exploratória pode utilizar como procedimento de coleta de dados o levantamento bibliográfico, para que os autores se familiarizem com o tempo, desta forma, utilizou-se pesquisa bibliográfica, com levantamento de informações previamente elaboradas e publicadas, a mesma foi realizada no período de agosto a novembro de 2015 .

Utilizou-se a base Ebsco para pesquisa e nesta base obteve-se um artigo utilizando a combinação "Hipótese de Porter", que foi excluído por não tratar sobre a Hipótese estudada e a combinação "Porter Hypothesis" resultou em 82 artigos publicados entre os anos de 2005 e 2015 de diferentes países. Posteriormente foi feita a análises, quantitativa, considerando:

a) volume de artigos publicados no período;

b) volume de artigos publicado por país;

c) evolução anual das publicações;

d) caracterização de autoria:

- Parcerias com instituições internacionais;

- Número de autores por artigo;

- Número de publicações por autores.

Por fim, foi realizada uma análise qualitativa dos artigos.

\section{RESULTADOS}

Conforme explicitado anteriormente, a análise será subdividida em duas partes, a primeira análise quantitativa e depois qualitativa.

\subsection{Análise quantitativa dos artigos}

Analisou-se 82 artigos da base de pesquisa Ebsco publicados entre 2005 e 2015, observa-se que o ano que teve o maior número de publicações sobre a Hipótese de Porter foi 2008 com 10 publicações, seguido por 2015 com 9, conforme demonstrado no Gráfico 1.

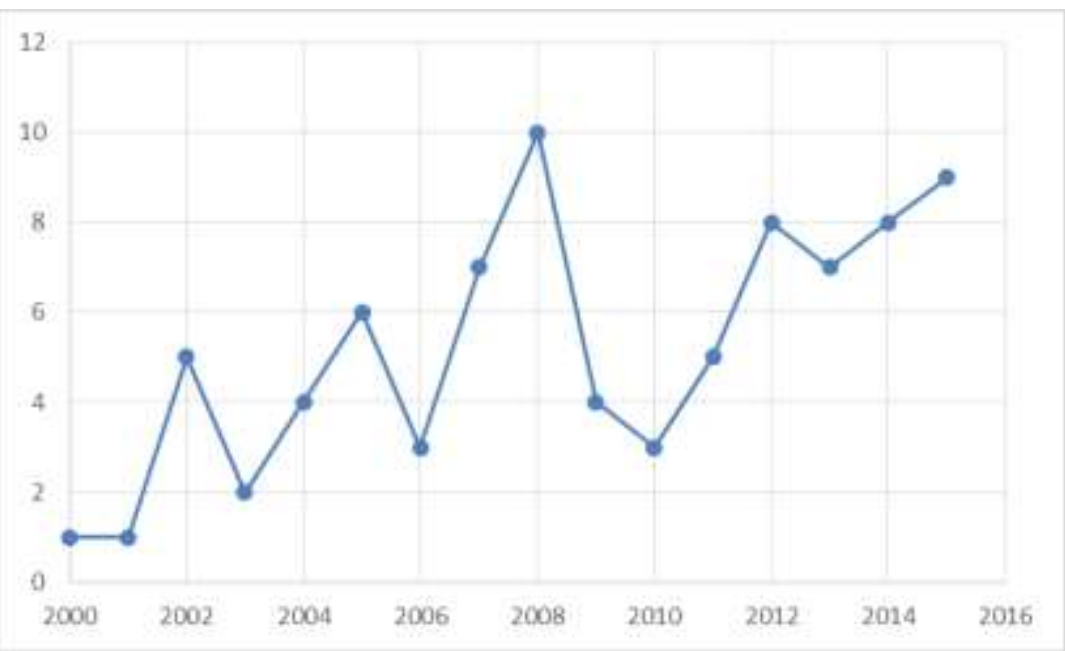

Figura 1. Volume de publicações anual

Fonte: Dados da pesquisa 
Quanto aos países dos pesquisadores obteve-se uma variação entre 23 países, com destaque para os Estados Unidos com 35 autores, seguido por Alemanha, demonstrado na Tabela 1.

Tabela 1

Origem dos pesquisadores

Total de autores

País

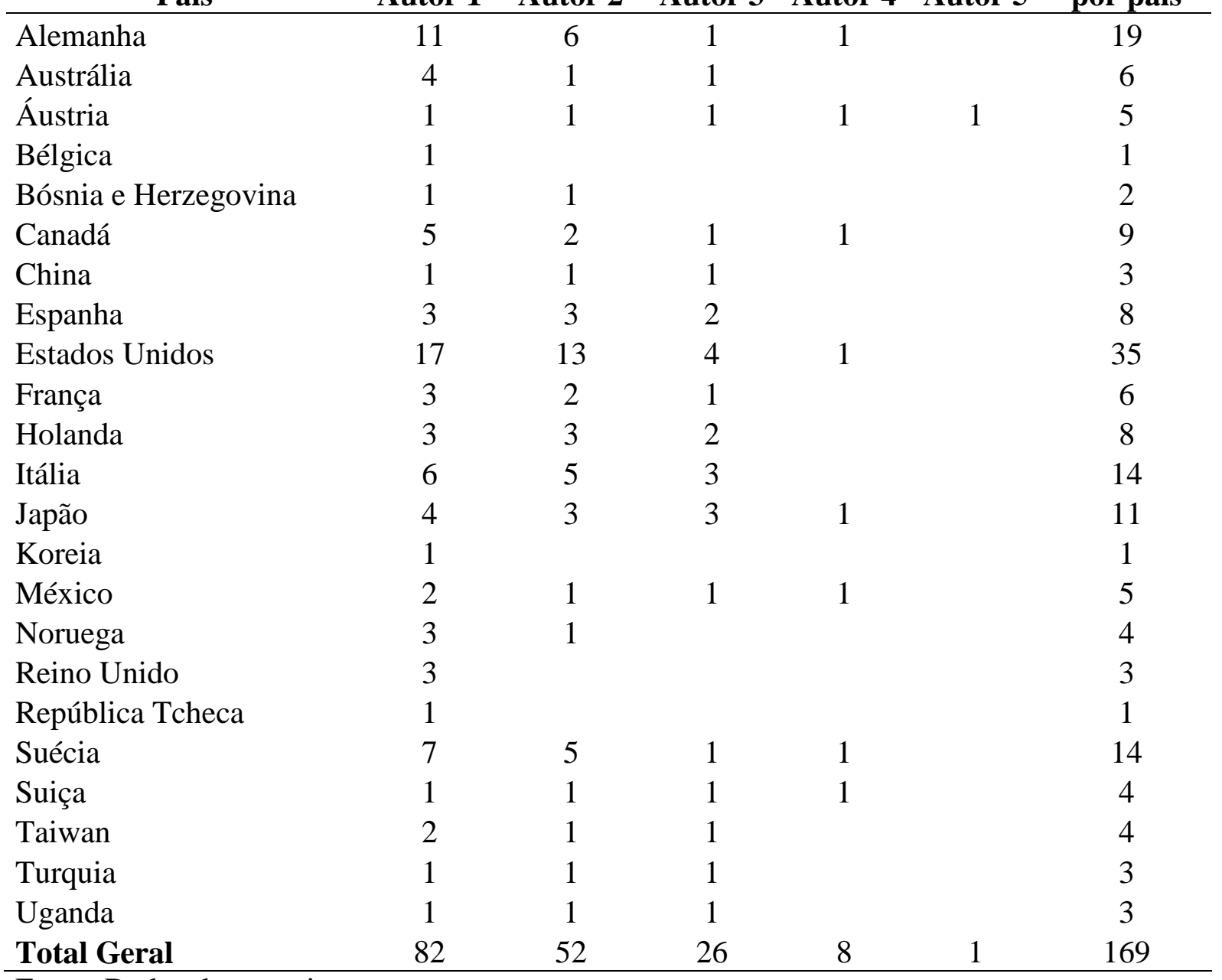

Fonte: Dados da pesquisa

Destaca-se que em $12 \%$ dos artigos os autores pertencem a dois ou mais países, demonstrando que há uma busca por companheiro de pesquisa em outras Instituições de Ensino e muitas vezes até de outros países. Quando comparado qual autor mais publicou sobre o assunto os Estados Unidos continuam em primeiro lugar com três autores sendo dois com 3 publicações e um com 2 publicações, conforme pode ser observado na Tabela 2. 
Tabela 2:

Frequência de autores

\begin{tabular}{llc}
\hline Autor & País & Quant. Publicações \\
\hline Ambec, Stefan & França & 3 \\
Barla, Philippe & Canadá & 2 \\
Constatini, Valeria & Itália & 2 \\
Earnhart, Dietrich & Estados Unidos & 3 \\
Greaker, Mads & Noruega & 3 \\
Lanoie, Paul & Canadá & 2 \\
Lundgren, Tommy & Suécia & 2 \\
Managi, Shunsuke & Japão & 2 \\
Mohr, Robert D. & Estados Unidos & 2 \\
Marklund, Per-Olov & Suécia & 2 \\
Rassier, Dylan G & Estados Unidos & 3 \\
Wackerbauer, Johann & Alemanha & 2 \\
Triebswetter, Ursula & Alemanha & 2 \\
\hline
\end{tabular}

Fonte: Dados da pesquisa

Obteve-se 55 periódicos diferentes, de diferentes países, com pelo menos uma publicação sobre a Hipótese de Porter. Destaca-se que o periódico que até 2015 tinha mais artigos relacionados a HP é a Journal of Environmental Economics \& Management, com oito publicações, seguido pelo Ecological Economics com seis publicações, Bussiness Strategy \& the Environment, European Environment: The Journal of European Environmental Policy (Wiley), Journal of Productivity Analysis, Land Economics, Research Policy, esource \& Energy Economics contavam com três publicações cada; os periódicos Energy Economics, Growth \& Change, Journal of Cleaner Production contavam com duas publicações e o restante com apenas uma publicação cada.

Com relação ao número de autores por artigo, $10 \%$ apresentaram quatro autores, $22 \%$ três autores, 35\% dois autores e 33\% apenas um autor, deve-se ressaltar que em nenhum dos artigos havia mais que quatro autores. Quanto as palavras chaves houve uma variação entre 2 a 6 por artigo, dessa forma, obteve-se 142 palavras distintas. Observa-se na Figura 2 representa uma nuvem de palavras desenvolvida por meio da ferramenta online World Cold, que a palavra mais citada nas palavras-chaves foi Porter Hypothesis (Hipótese de Porter), Environmental Regulation (regulamentação ambiental) e Innovation (inovação). 


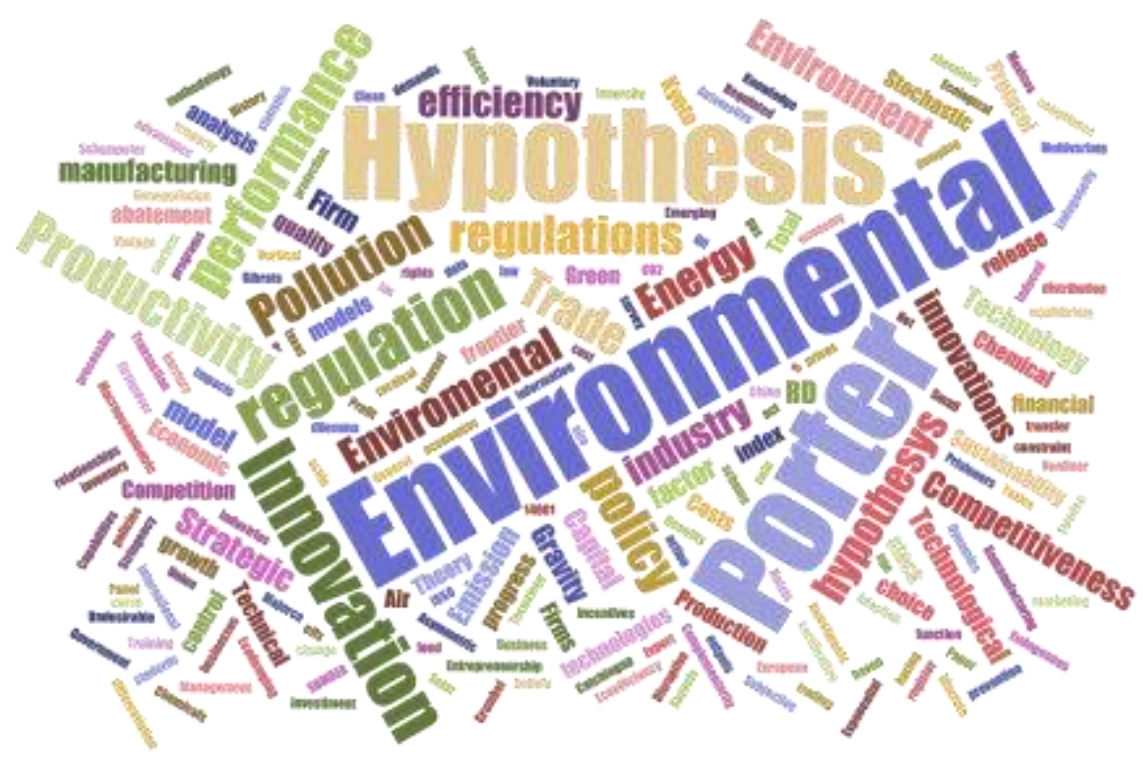

Figura 2. Nuvem de palavras

Fonte: Dados da pesquisa

\subsection{Análise qualitativa dos artigos}

Desde sua primeira publicação em 1991 por Porter e posteriormente em 1995 por Porter e Van der Linde a Hipótese de Porter (HP) tem sido testada por autores de diversos países, que tentam provar sua eficiência ou não na competitividade empresarial, conforme foi exposto nesta seção.

Smith e Ayerbe (2000) ao realizarem testes experimentais da HP concluiram que as regulamentações ambientais criam compensações de inovações que de outra forma não seriam realizadas. Entretanto, Mohr (2002) afirma que uma política ambiental que produz resultados compatíveis com a HP não é necessariamente ideal, pois essa política pode induzir as empresas a experimentarem novas tecnologias, mas também pode criar custos a curto prazo.

Ao analisarem o setor de processamento de alimentos dos EUA e do México Alpay, Buccola e Kerkvliet (2002), concluíram que as normas de poluição não tiveram nenhum impacto sobre a rentabilidade ou produtividade dos EUA. Entretanto, no México a rápida elevação dos padrões ambientais reforçaram o crescimento da produtividade dos processadores de alimentos, portanto, os autores afirmam que a eficiência da HP, depende do território, pois o mesmo setor foi estudado em dois países e enquanto em um houve resultados positivos no outro não fez diferença.

Ambec e Barla (2002) e Feichtinger, Hartl, Kort e Veliov (2005), após investigarem o efeito da política ambiental sobre a composição do capital, concluíram que a política ambiental mais rigorosa acaba causando efeito negativo sobre os lucros da indústria, contrariando, assim, a HP.

Schluga (2003) ao explorar se as normas tiveram algum impacto na competitividade dos fabricantes austríacos de tintas, revestimentos e adesivos, concluiu que as regulamentações mais rígidas não influenciam nem negativa e nem negativamente na competitividade dos pesquisados. No entanto, o autor evidenciou que os esforços para cumprir com a legislação resultaram em novas ideias e permitiu que algumas empresas adquirissem novas competências e tecnologias que não adquiririam na ausência da regulamentação.

Connelly e Limpaphayom (2004) pesquisaram empiricamente o Thailand Institute of Directors' Corporate Governance Brenchmarking Survey e constataram que não há relação significativa entre os relatórios ambientais e o desempenho de contabilidade, o que sugere que a

Revista de Direito Brasileira | São Paulo, SP | v. 18 | n. 7 | p. 282 - 300 | Set./Dez. 2017 
divulgação de boas políticas ambientais não afetam a rentabilidade a curto prazo. No entanto, existe uma relação positiva e significativa, não-linear entre os relatórios ambientais e avaliação de mercado. Demonstrando que as políticas ambientais afetam o desempenho no longo prazo, podendo, assim aumentar a competitividade e maximizar a riqueza dos acionistas.

Frohwein e Hansjürgens (2005), afirmaram que a HP funciona bem em termos de incentivo a inovações para aumentar a produtividade dos recursos, porém em certos segmentos da indústria de produtos químicos europeia apresentou efeitos negativos sobre a concorrência e inovação, ou seja, o seu desempenho depende do setor.

Managi, Opaluch, Di e Grigalunas (2005) estudaram a indústria de gás e petróleo e por meio de simulações que objetivaram medir vários componentes da produtividade total dos fatores dentro de um modelo de produção conjunto, que considera tanto saídas ambientais e de mercado e demonstraram que, em geral, a política ambiental tem pouco efeito sobre a participação no mercado de exportação, pois apesar da melhoria da tecnologia da produtividade do meio ambiente, a produtividade ambiental fica aquém das saídas de mercado.

Popp (2005) realizou simulações por meio de um modelo de pesquisa e desenvolvimento, para analisar se havia retorno financeiro após a adequação ambiental e concluiu que de 8 a 24 por cento resultou em casos de lucros pós regulamentação superior aos lucros pré regulamentação.

Cerin (2006) assevera que as empresas somente agiriam de maneira altruísta para o bem da natureza se houvesse situações vantajosas dentro do seu próprio resultado financeiro, especialmente se os consumidores promoverem tais características, ou seja, os consumidores devem cobrar tais ações das empresas e também cobrar o Governo para que o mesmo crie regulamentações.

Greaker (2006) ao analisar o mercado de inovações tecnológicas, concluiu que há resistência das empresas com relação à regulamentação ambiental rigorosa, porque o lucro pode diminuir, mesmo que a competitividade melhore, pois, apesar da diminuição do custo marginal é possível que haja um aumento no custo total, uma vez que uma cota menor de emissão implica em aquisição de novos equipamentos.

Hamamoto (2006) ao investigar as questões relativas aos efeitos do rigor das regulamentações sobre a inovação e produtividade das indústrias japonesas, apontou que as despesas de controle da poluição têm uma relação positiva com as despesas com P\&D e têm uma relação negativa com a idade média do estoque de capital. $\mathrm{O}$ aumento do investimento com $\mathrm{P} \& \mathrm{D}$ estimulado pelo rigor regulamentar tem um efeito positivo significativo sobre a taxa de crescimento da produtividade total dos fatores.

Burnett, Hansen e Quintana (2007) afirmam que a poluição é uma forma de ineficiência técnica e que os regulamentos devidamente concebidos estimulam as empresas a desenvolver inovações de controle de poluição. No entanto, requer esforço coordenado por parte das empresas e legisladores para entender as necessidades de cada um e oferecer alternativas que proporcionarão benefícios para cada uma das partes.

Após desenvolverem uma análise longitudinal de pequenas e médias empresas de horticultura holandesa, sob diferentes regimes de política ambiental, Vlist, Withagen e Folmer (2007) afirmaram que a regulamentação ambiental mais restrita reduz a ineficiência técnica, apoiando a HP.

Ao pesquisar se a região na qual a empresa está instalada influencia na sua capacidade tecnológica do ponto de vista de aglomeração industrial e externalidades dinâmicas em Taiwan, Chen (2007) concluiu que nos locais que as regulamentações ambientais eram mais rígidas os aglomerados industriais apresentavam uma busca maior por novas tecnologias de produção, aumentando assim a sua qualidade, produtividade e competitividade.

Todavia, Oberndorfer e Rennings (2007) por meio de pesquisa realizada com intuito de avaliar impacto das legislações ambientais na UE sobre os custos e competitividade, concluiram 
que há influência negativa da política ambiental, os autores asseguram que os resultados são melhores, quando a empresa realiza alguma ação voluntariamente.

Bates e Robb (2008) afirmam que a eficiência da HP depende do local ao qual a empresa está inserida, pois o nicho minoritário de bairros não oferecem as empresas jovens um conjunto atraente de oportunidades, portanto a sua viabilidade comercial é menor, quando relacionado ao nicho de mercado regional mais amplo.

Costantini e Crespi (2008) analisaram evidências empíricas sobre a evolução dos fluxos de exportações de tecnologias ambientais em diferentes países para o setor energético, em particular na UE e argumentaram que a regulação ambiental representa uma fonte significativa de vantagens competitivas, pois o rigor dessas regulamentações complementada pela força do Sistema Nacional de Inovação apresenta importante desempenho nas exportações no domínio das tecnologias energéticas.

Greaker e Rosendahl (2008) analisaram se uma política ambiental rigorosa influência positivamente na indústria de exportação e se esta política pode ser utilizada para aumentar a concorrência no setor upstream e averiguaram que uma política ambiental rigorosa não gera resultados positivos no que diz respeito ao desenvolvimento bem sucedido de novos setores de exportação com base em tecnologia de redução.

Em sua pesquisa Horbach (2008) utilizou duas bases de dados do painel alemão, o painel de criação do Instituto de Pesquisa de Emprego (IAB) e Mannheim painel de Inovação (MIP) do Centro de Inovação Econômica Europeia (ZEW), para explorar as determinantes do meio ambiente. Por meio de estimativas econométricas, o autor demonstrou melhoria das capacidades tecnológicas por P\&D desencadeando inovações ambientais. Ainda demonstrou que a regulamentação ambiental, como instrumento de gestão ambiental e de mudança organizacional geral, também incentiva a inovação ambiental, corroborando com a HP.

Lanoie, Patry e Lajeunesse (2008) a partir de análise empírica da relação entre o rigor da regulamentação ambiental e da produtividade total dos fatores no crescimento do setor manufatureiro de Quebec, concluíram que o impacto contemporâneo da regulamentação ambiental sobre a produtividade é negativo, o resultado oposto é observado com variáveis reguladoras defasadas, o que é consistente com a HP e o efeito é mais forte em um subgrupo de indústrias que estão mais expostos à concorrência internacional.

Lindmark e Bergquist (2008) examinaram a evolução histórica do processo de adaptação ambiental em uma empresa de fundição de metais sueco, contrastando o resultado com casos no Canadá. As descobertas sugerem que o sistema sueco na exclusão de partes interessadas, com foco em emissões de plantas e estipulando a redução da poluição a custos econômicos viáveis. Isto permitiu uma estratégia de "expansão por redução" de emissões. Mas os autores afirmam que os resultados podem variar de acordo com o local no qual a empresa está instalada.

Para Mohr e Saha (2008) a HP é inconsistente como modelo econômico ou simplesmente como medição de custos, pois por meio de pesquisa, os autores concluíram que mesmo sem inovarem as empresas podem lucrar tanto quanto as que inovam.

Triebswetter e Wackerbauer (2008a) ao desenvolverem um estudo de caso regional sobre o impacto de inovações de produtos ambientalmente corretos sobre a competitividade do setor automotivo, ferroviário e comercial de veículos no sul da Alemanha, concluíram que a legislação estimula a inovação e leva a situações "ganha-ganha", com a redução simultânea de poluição e aumento da produtividade.

Triebswetter e Wackerbauer (2008b) examinaram o impacto das inovações ambientais na competitividade das empresas tanto da indústria, do ambiente e setores afins na região de Munique e argumentaram que inovação ambiental é conduzida por uma mistura de fatores internos e externos à empresa: não só pressão regulatória, mas também de custos, vantagens competitivas, liderança tecnológica e pressão dos clientes são fatores importantes. Inovações impulsionadas por regulamentações contribuem para o desempenho competitivo das empresas da 
amostra de uma maneira semelhante como inovações ambientais que são realizadas voluntariamente. Isso produz prova para a HP, que estimula a inovação e leva a "ganha-ganha", a poluição é reduzida e a produtividade aumenta.

André, González e Porteiro (2009) utilizando o equilíbrio de Nash afirmaram que a implantação de uma "política verde" pode melhorar a qualidade ambiental do produto, aumentando simultaneamente os lucros das empresas. Destacaram ainda que a regulamentação ambiental não só pode tornar as empresas mais rentáveis, mas, também, pode aumentar o número de consumidores.

Kriechel e Ziesemer (2009) analisaram a HP por meio de um jogo de cronometragem de adoção de tecnologia, pelas simulações no jogo, chegaram à conclusão de que a HP faz com as empresas façam investimentos antecipados em tecnologias e isso acaba levando a um desequilíbrio, uma vez que a aquisição tardia, segundo o jogo, traz o equilíbrio e proporciona mais lucro.

López-Gamero, Claver-Cortés e Molina-Azorín (2009) realizaram em um primeiro momento um estudo comparativo de oito empresas espanholas, com as proposições que emergiram nesta primeira fase foram testadas por meio de um modelo de equações estruturais 239 hoteis e 208 empresas afetadas pela lei do Painel Intergovernamental sobre Mudanças do Clima - IPCC na Espanha. Com os resultados obtidos os autores não validaram nem refutaram a HP, eles argumentaram que tanto se houver maciça margem de melhorias, as empresas só precisam estar cientes de que mesmo não tendo uma regulamentação ambiental real, elas precisam reconhecer o seu papel a ser desempenhado pelo ambiente natural.

Brännlund e Lundgren (2010) ao investigarem o feito de um imposto sobre o CO2 na lucratividade em dados sobre a produção de insumos da indústria sueca entre 1990-2004, postularam que os resultados indicam que há evidências de um efeito Porter "invertido" na maioria dos setores industriais especialmente em indústrias de energia intensiva.

Rassier e Earnhart (2010) testaram a versão "strong” da HP na regulamentação da água em empresas produtoras de produtos químicos, concluíram que quanto mais apertada a regulamentação menor será a rentabilidade no setor, pois o haverá aumento nos custos, implicando em uma redução de cerca de $0,8 \%$ anual no retorno sobre as vendas.

Rennings e Rammer (2010) analisaram se a inovação ambiental impulsionada pela regulamentação era semelhante ao grau de inovação quando comparado com outros tipos de produtos e inovações de processos e qual o impacto dessa regulamentação no sucesso da empresa. Os regulamentos em favor de uma mobilidade sustentável contribuíram para maiores vendas com novidades, enquanto os regulamentos no domínio da gestão da água diminuíram o sucesso na inovação. No que diz respeito à margem de custo de uma empresa, novos processos implementados para cumprir os requisitos regulamentares ambientais incidiram em menor rentabilidade, indicando custos mais elevados para este tipo de inovação que não pode ser passado para os preços de venda.

Bernard (2011) em seu modelo teórico de remanufatura apoia a HP, pois acredita que a hipótese ao estimular as indústrias a respeitarem as normas ambientais, ainda pode influenciar positivamente em sua produtividade e consequentemente em sua competitividade.

Lanoie, Laurent-Luccheti, Johnstone e Ambec (2011) realizaram a pesquisa baseada nas três distintas demonstrações sobre a eficiência da HP de Jaffe e Palmer (1997), (narrow - uma regulamentação ambiental flexível aumenta os incentivos à inovação empresarial; weak - efeitos positivos da regulamentação ambiental, mesmo quando as inovações são pelo custo de oportunidade e strong - a regulamento compensaria os custos adicionais e aumentariam a competitividade e produtividade), segundo os mesmos há incentivo apenas para as duas primeiras demonstrações. Há grande apoio para a versão "weak", este apoio encontra-se na política ambiental que induz a inovação, há ainda apoio para a "narrow", pois são mais flexíveis e induzem a normas de base tecnológica e não há suporte para a "strong", pois os autores afirmam 
que a inovação compensa, apenas, parcialmente os custos do cumprimento das políticas ambientais.

Liu, Dai e Cheng (2011) analisaram os motores da inovação ambiental e seus respectivos efeitos sobre a competitividade das empresas com base em 37 entrevistas com 18 empresas chinesas. Concluíram que a inovação ambiental é conduzida por uma mistura de fatores de regulamentação ambiental, pressão de custos, vantagens competitivas e pressão dos clientes, rendendo prova para a HP, que assume que a regulamentação ambiental estimula a inovação e leva à oportunidade que simultaneamente, reduz a poluição e aumenta a competitividade

Rassier e Earnhart (2011) ao realizarem uma pesquisa específica a curto e longo prazo no desempenho financeiro sobre uma regulamentação ambiental denominada "ação água boa", que tinha por objetivo o exame do efeito de atividades rotineiras do dia a dia que requerem o uso de água, averiguaram que a regulamentação melhora o desempenho financeiro tanto no curto e no longo prazo, com um efeito mais forte no longo prazo. Estes resultados sugerem que alguns benefícios líquidos podem ser realizados durante a transição de curto prazo para cumprir um limite de descargas permitida mais apertada, com os benefícios adicionais resultantes para empresa no longo prazo, porque a empresa tem mais tempo para inovar.

Costantini e Mazzanti (2012) ao realizarem investigação sobre como a competitividade das exportações da União Europeia eram afetadas pela regulamentação e inovação ambiental, concluíram que as políticas públicas e os padrões de inovações privadas, tanto desencadeiam uma maior eficiência do processo de produção através de vários mecanismos de complementaridade, transformando assim a percepção de ações de proteção ambiental, como um custo de produção em um benefício líquido.

Horváthová (2012) realizou exame do efeito intertemporal do desempenho ambiental no desempenho financeiro, os resultados sugerem que no primeiro ano o desempenho financeiro é negativo, mas a partir do segundo torna-se positivo. Como consequência, os resultados indicam que a HP é para longo prazo.

Kataria (2012) realizou uma pesquisa empírica, utilizando teorias de psicologia de viés cognitivo, em uma amostra de estudantes de economia e ciência para analisar se certas circunstâncias, de preferência uma relação positiva, gera uma expectativa que é realmente o esperado. A conclusão foi que os estudantes que se preocupavam mais com questões ambientais eram mais propensos a acreditar na HP, existindo, assim, uma correlação entre as posições políticas dos economistas e os seus valores ideológicos.

Yang, Tseng e Chen (2012) ao analisarem dados de nível da indústria taiwanesa de 1997 a 2003, perceberam que as taxas de redução da poluição, estavam positivamente relacionadas com os regulamentos ambientais, por outro lado, as despesas de capital com a redução da poluição não tinham influência estatística significativa em P\&D. Avaliando a influência da indução de $\mathrm{P} \& \mathrm{D}$ pela legislação ambiental na produção industrial, aprontaram que havia relação significativa entre eles, concluindo que as regulamentações ambientais mais rigorosas podem aumentar a competitividade industrial.

Yang e Yao (2012) avaliaram o impacto da conformidade ambiental na inovação das empresas e desempenho financeiro, utilizando um painel de dados de empresas chinesas entre os períodos de 2000-2005. Os resultados demonstraram que a certificação ISO 14000 tem um impacto significativamente positivo sobre a rentabilidade empresarial. Além disso, as empresas certificadas apresentaram maiores volumes de vendas por trabalhador e quotas de mercado no país, o que sugere um mecanismo alternativo para a HP no contexto de uma economia em desenvolvimento.

Antonioli, Mancinelli e Mazzanti (2013) ao investigar se a aplicação conjunta de inovação e treinamento organizacional das empresas promovem a sua adoção de inovação ambiental e se essa correlação é compatível com a HP, postularam que a HP só é verdadeira 
quando a amostra é restrita a setores mais poluentes e regulamentados, no entanto, a regulamentação rigorosa não é o único fator responsável por todas as inovações ambientais.

Broberg, Marklund, Samakovlis e Hammar (2013) após avaliar os efeitos estáticos e dinâmicos da política ambiental sobre a produtividade, rejeitaram a HP, pois afirmam que há fraca relação entre investimentos em proteção ambiental e eficiência técnica. Os autores consideram que a principal motivação para estimular as empresas a protegerem o meio ambiente não é para aumentar a eficiência da empresa, mas para diminuir os danos ambientais provenientes de falhas de mercado.

Inoue, Arimura e Nakano (2013) examinaram os fatores que podem induzir a inovação tecnológica ambiental, considerando a ISO 14001, uma abordagem voluntária a gestão ambiental e analisando como a proficiência e/ou maturidade nesta regulamentação influência a P\&D ambiental na indústria japonesa. Os resultados ofereceram evidências empíricas de que o nível de maturidade da ISO 14001 influencia positivamente os investimentos em P\&D ambiental, umas vez que as empresas estão mais propensas a gastar mais com melhorias.

Kózluk e Zipperer (2013) desenvolveram uma revisão das evidências empíricas sobre a relação entre o rigor da política ambiental, o crescimento da produtividade e os vários canais pelos quais tais efeitos podem ter lugar e concluíram que os resultados são ambíguos, muitos resultados são frágeis e de contexto específico, impedindo a generalização de conclusões, problemas práticos relacionados com dados, estratégias de medição são discutidos, levando a sugestões de como eles podem ser resolvidos em pesquisas futuras. Estes incluem: melhorar a medição do rigor da política ambiental; investigar sobre os efeitos de diferentes tipos de instrumentos e detalhes de desenhos de instrumentos; explorar as variações e a utilização complementar de diferentes níveis de agregação.

Em seu artigo Ziesemer (2013) argumenta que a HP carece de um fundamento microeconômico abrangente, uma vez que não considera as propriedades e ramificações do capital do conhecimento. Para suprir esta deficiência o autor desenvolve uma visão considerando o capital do conhecimento como o ativo mais importante na obtenção de uma competitividade sustentável.

Doganay, Sayed e Taskin (2014) realizaram um estudo empírico objetivando analisar a eficiência ambiental de 111 países de 1980-2009, e constataram que os países que melhoraram sua eficiência ambiental puderam experimentar fortes efeitos de comércio internacional, por meio do aumento das exportações e importações. Enquanto a relação positiva entre a melhoria da eficiência e as exportações apoiam a HP, a ligação positiva entre melhorias de eficiência e importações é de suporte de fortes efeitos sobre os rendimentos positivos por conta dos esforços ambientais. Estes resultados são significativos para estratégias de estimação alternativas, pois apoiam aos esforços globais para melhorar a eficiência ambiental dos países.

Ford, Steen e Verreynne (2014), analisaram o impacto da regulamentação ambiental sobre a inovação no nível top-down (tenta medir a resposta a inovação) e bottom-up (as empresas produzem inovações que excedem os níveis de conformidade como uma estratégia competitiva) em empresas de petróleo e gás australianas. Evidenciaram que grandes empresas tendem a ter alguma resistência às regulamentações ambientais, mas apoiam o aumento da amplitude tecnológica, por acreditarem que assim ganham vantagem sobre os concorrentes. Ou seja, as inovações das grandes empresas são impulsionadas pelas vantagens competitivas, não pelas questões ambientais.

Groba (2014) realizou uma investigação empírica para identificar as diferenças tecnológicas entre as diferentes energias renováveis e identificar a extensão do comércio de tecnologias de energia como instrumento de transferência de tecnologia. Os resultados demonstraram que a Europa é um mercado com potencial crescimento para o consumo de energias renováveis, e a regulamentação ambiental determina forte desempenho nas exportações, 
uma vez que, os países estão fortemente comprometidos por meios de gastos e suporte de energia solar.

Lim e Prakash (2014) examinaram um painel de 79 países no período de 1996-2009, objetivando explorar a ligação da regulamentação na inovação no contexto de caráter voluntário. Eles identificaram que o nível de participação na ISO 14001 é um preditor significativo de pedidos de patentes ambientais de um país. De acordo com os autores os gestores públicos devem considerar regulamentos voluntários sobre a inovação, além de seus efeitos de primeira ordem sobre a poluição e conformidade regulamentar.

Sadeghzadeh (2014) investigou a influência das políticas ambientais sobre a concorrência no mercados, os resultados foram consistentes com a HP, no sentido de que as normas ambientais motivam tecnologias de redução, e sua adoção melhora a produtividade e qualidade ambiental. No entanto, o ganho na produtividade é impulsionado principalmente pela realocação de recursos entre as empresas, pois de acordo com o autor, regulamentações mais rígidas prejudicam o ambiente competitivo pelo aumento dos preços médios e concentração do mercado, o bem-estar social também cai por causa da ausência de uma forte concorrência.

Stella, Aggrey e Eseza (2014) investigaram a taxa de crescimento do setor industrial da Uganda e concluíram por meio de regressão que apenas as empresas de médio porte apresentam crescimento significativo com regulamentações ambientais mais rígidas, enquanto que as pequenas e grandes empresas não apresentam diferença significativa.

Franckx (2015) desenvolveu uma revisão teórica das evidências disponíveis sobre a relevância da HP nas normas de emissão de automóveis. A literatura confirma que os padrões de emissões para os automóveis tiveram efeitos importantes sobre a inovação (a forma "weak" da hipótese de Porter). No entanto, não há nenhuma evidência de que as regulamentações levam a um aumento global na produtividade (a versão "strong" da hipótese Porter). De acordo com o autor, regulamentações rigorosas de emissão podem fazer com que os países tenham vantagens competitivas, por se tornarem líderes. Por exemplo, os países estão mais propensos a ter padrões de emissão para veículos nacionais mais rigorosas se exportar mais automóveis e componentes de automóveis para países que têm padrões veiculares mais rígidos. Há também evidências de que os países que recebem mais investimento estrangeiro direto no setor automóvel são mais propensos a ter normas mais rigorosas de emissões domésticas.

Lundgren e Marklund (2015) afirmam que o desempenho ambiental induzido pela política ambiental não é determinante do desempenho econômico, enquanto que o desempenho ambiental voluntário ou impulsionado pelo mercado apresentam efeitos significativos e positivos na eficiência econômica.

Rassier e Earnhart (2015) investigaram os efeitos da regulamentação ambiental sobre a rentabilidade real e prevista no setor de tratamento de água. A rentabilidade efetiva apresentou consistência com a HP, entretanto a rentabilidade esperada foi superior ao valor real. Assim, percebe-se que os investidores, parecem não valorizar os efeitos positivos de uma regulamentação mais rigorosa.

Razumova, Ibáñez e Rey (2015) utilizaram um banco de dados de hotéis de Majorca para analisar os vários determinantes de inovações tecnológicas derivados da HP, não testados nos hotéis. Os resultados demonstraram que a predisposição geral de implantarem inovações, parte de um gerente com remuneração baseada no desempenho ambiental, satisfação pessoal, contabilidade ambiental e treinamentos. Além disso, o uso de sistemas de gestão ambiental está associado a inovações com potencial redução de custos, enquanto que hotéis com mais estrelas estão associados a inovações que contribuem para a qualidade do hotel, como tratamento de águas residuais, redução e isolamento de ruídos, melhorias do impacto visual.

Rubashkina, Galeotti e Verdolini (2015) realizaram uma investigação empírica dos pontos fortes e fracos da HP no setor industrial de 17 países europeus, entre 1997 e 2009 . Os autores encontraram evidências de impacto positivo da regulamentação ambiental quando 
considerado à inovação das empresas estudadas, porém quando considerado o grau de controle de poluição e esforço para reeducação ambiental, não encontraram nenhuma evidência a favor da HP.

Percebe-se que alguns corroboram com a HP, afirmando que regulamentações ambientais mais rígidas impulsionam a inovação, consequentemente a concorrência, porém alguns apresentam resultados diferentes, afirmando que as regulamentações ambientais não causam os efeitos esperados, ou que quando as ações ambientais são realizadas voluntariamente pelas empresas seus resultados são melhores.

Um número significativo de pesquisadores, pontuam ser relevante que as legislações ambientais versem, incisivamente, sobre a necessidade emergencial da composição e comercialização de produtos que não prejudiquem ou que minimizem ao máximo os danos cometidos ao meio ambiente, uma vez que consideram esta estratégia mais eficiente do que incentivar a investir em melhorar o que já está poluído.

\section{CONCLUSÃO}

As opiniões sobre a validade ou não da HP divergem muito nos estudos empíricos, porém a maioria acredita que ela cumpre com o seu objetivo, que é estimular a competitividade e inovação tecnológica por meio de uma rígida regulamentação ambiental.

Destaca-se que foi possível identificar nos estudos, mesmo naqueles que não corroboram com a HP, que sem as regulamentações ambientais não haveria investimento suficiente por parte das empresas na preservação e não poluição do meio ambiente.

Percebe-se ainda que há grande divergência de resultados quanto aos setores e regiões aplicadas, sugerindo que a eficiência da HP depende do local no qual a empresa está inserida. Para estudos futuros, identifica-se a possibilidade de pesquisar outros autores que discutam a competitividade sustentável ou sustentabilidade competitiva e ainda desenvolver uma pesquisa sobre a HP que seja aplicável em todos os setores.

\section{REFERÊNCIAS BIBLIOGRÁFICAS}

ALPAY, E.; BUCCOLA, S.; KERKVLIET, J. Productivity growth and environmental regulation in Mexican and U.S. food manufacturing. American Journal of Agricultural Economics, v. 84, n.4, 887-901, 2002.

AMBEC, S.; BARLA, P. A theoretical foundation of the Porter hypothesis. Economics Letters, n. 75, p. 355-360, 2002.

AMBEC, S.; COHEN, M. A.; ELGIE, S.; LANOIE, P. The Porter Hypothesis at 20: con environmental regulation enhance innovation and competitiveness? Environmental Economics and Policy. v. 7 n. 1 p. 2-22, jan., 2013.

ANDRÉ, F. J.; GONZÁLEZ P.; PORTEIRO, N. Strategic quality competition and the Porter Hypothesis. Journal of Environmental Economics and Management, v. 57, p. 182-194, 2009.

ANSANELLI, S. L. de M. Exigências ambientais europeias: novos desafios competitivos para o complexo eletrônico brasileiro. Revista Brasileira de Inovação, v. 10, n. 1, jan./jun., 2011. 
ANTONIOLI, D.; MANCINELLI, S.; MAZZANTI, M. Is environmental innovation embedded within high-performance organizational changes? The role of human resource management and complementarity in green business strategies. Research Policy, v. 42, p. 975-988, 2013.

AQUINO, S. R. F. de; CAVAlHEIRO, L. P. R.; PELLENZ, M. A tutela jurídica da água no Brasil: reflexões a partir dos direitos da natureza. Revista de Direito Brasileira - RDB, São Paulo, v. 14, n. 6, p. 65-79, maio/ago., 2016.

ASHFORD, N. A. Na innovation-based strategy for a sustainable environment. In: J. Hemmelskamp, K. Rennings, F. Leone (org.). Innovation-oriented environmental regulation: theoretical approach and empirical analysis. New York: ZEW Economic Studies, 2000, p. 67107.

BATES, T.; ROBB, A. Analysis of young neighnorhood firms serving urban minority clients. Journal of Economics and Business, v. 60, p. 139-148, 2008.

BERNARD, S. Remanufacturing. Journal of Environmental Economics and Management, v. 62, n. 3, p. 337-351, 2011.

BRÄNNLUND, R.; LUNDGREN, T. Environmental policy and profitability: evidence from Swedish industry. Environmental Economics and Policy Studies, v. 12, p. 59-79, 2010.

BROBERG, T.; MARKLUND, Per-Olov; SAMAKOVLIS, E.; HAMMAR, H. Testing the Porter hypothesis: the effects of environmental investments on efficiency in Swedish industry. Journal of Productivity Analysis, v. 40, p. 43-56, jan., 2013.

BURNETT, R. D.; HANSEN, D. R.; QUINTANA, O. Eco-efficiency: achieving productivity improvements through environmental cost management. Accounting and the Public Interest, v. 7, n. 1, p. 66-92, 2007.

CALIENDO, P.; MUNIZ, V. Política fiscal e desenvolvimento tecnológico-empresarial: uma análise crítica sobre inovação e tributação. Revista de Direito Brasileira - RDB, São Paulo, v.12, n. 5, p. 155-172, 2015.

CERIN, P. Bringing economic opportunity into line with environmental influence: a discussion on the Coase theorem and the Porter and van der Linde hypothesis. Ecological Economics, v. 56, p. 209-225, 2006.

COLlinS, J.; HUSSEY, R. Pesquisa em administração: um guia prático para alunos de graduação e pós-graduação. 2. ed. Porto Alegre: Bookman, 2005.

CONNELLY, J.; LIMPAPHAYOM, P. Environmental reporting and firm performance: evidence from Thailand. Journal of Corporate Citizenship, v. 13, p. 137-149, 2004.

COSTANTINI, V.; CRESPI, F. Environmental regulation and the export dynamics of energy technologies. Ecological Economics, v. 68, p. 447-460, 2008.

COSTANTINI, V.; MAZZANTI, M. On the green and innovative side of trade competitiveness? The impact of environmental policies and innovation on EU exports. Research Policy, v. 41, n.1, p. 132-153, 2012. 
DOGANAY, S. M.; SAYED, S.; TASKIN, F. Is environmental efficiency trade inducing or trade hindering? Energy Economics, v. 44, p. 340-349, 2014.

FEICHTINGER, G.; HARTL, R. F.; KORT, P. M.; VELIOV, V. M. Environmental policy, the Porter hypothesis and the composition of capital: effects of learning and technological progress. Journal of Environmental Economics and Management, v. 50, p. 434-446, 2005.

FORD, J. A.; STEEN, J.; VERREYNNE, M.-L. How environmental regulations affect innovation in the Australian oil and gas industry: going beyond the Porter Hypothesis. Journal of Cleaner Production, v. 84, p. 204-2013, 2014.

FRANCKX, L. Regulatory emission limits for cars and the Porter Hypothesis: a survey of the literature. Transport Reviews, v. 35, n. 6, p. 746-766, 2015.

FROHWEIN, T.; HANSJÜRGENS, B. Chemicals regulation and the Porter hypothesis: a critical review of the new European chemicals regulation. Journal of Business Chemistry, v. 2, n. 1, p. 19-36, jan., 2005.

GILL, A. C. Como elaborar projetos de pesquisa. 4. ed. São Paulo: Atlas, 2008.

GREAKER, M. Spillovers in the development of new pollution abatement technology: a new look at the Porter-hypothesis. Journal of Environmental Economics and Management, v. 52, p. 411-420, 2006.

GROBA, F. Determinants of trade with solar energy technology components: evidence on the Porter Hypothesis? Applied Economics, v. 46, n. 5, p. 503-526, 2014.

HAMAMOTO, M. Environmental regulation and the productivity of Japanese manufacturing industries. Resource and Energy Economics, v. 28, p. 299-312, 2006.

HORBACH, J. Determinants of environmental innovation - new evidence from German panel data sources. Research Policy, v. 37, n.1, p. 163-173, 2008.

HORVÁTHOVÁ, E. The impact of environmental performance on firm performance: short-term costs and long-term benefits. Ecological Economics, v. 84, p. 91-97, 2012.

INOUE, E.; ARIMURA, T. H.; NAKANO, M. A new insight into environmental innovation: does the maturity of environmental management systems matter? Ecological Economics, v. 94, p. 156-163, 2013.

JAFFE, A.B.; PALMER, K. Environmental regulation and innovation: a panel data study. Economics and Statistics, v. 79, p. 610-619, 1997.

KATARIA, M. The role of preferences in disagreements over scientific hypothesis: evidence on cognitive bias in formation of beliefs. The Journal of Socio-Economics, v. 41, n. 4, p. 364-369, 2012.

KÓZLUK, T.; ZIPPERER, V. Environmental policies and productivity growth - a critical review of empirical findings. OECD Journal: Economic Studies, v. 88, p. 1-37, nov., 2013. 
KRIECHEL, B.; ZIESEMER, T. The environmental Porter Hypothesis: theory, evidence and a model of timing of adoption. Economics of Innovation \& New Technology, v. 18, n.3, p. 267-294, 2009.

LANOIE, P.; PATRY, M.; LAJEUNESSE, R. Environmental regulation and productivity: testing the Porter hypothesis. Journal of Poductivity Analysis, v. 30, p. 121-128, 2008.

LANOIE, P.; LAURENT-LUCCHETI, J.; JOHNSTONE, N.; AMBEC, S. Environmental policy, innovation and performance: new insights on the Porter hypothesis. Journal of Economics \& Management Strategy, v. 20, n. 3, p. 803-842, 2011.

LIM, S.; PRAKASH, A. Voluntary regulations and innovation: the case of ISO 14001. Public Administration Review, v. 74, n. 2, p. 233-244, 2014.

LINDMARK, M.; BERGQUIST, A. K. Expansion for pollution reduction? Environmental adaptation of a Swedish and a Canadian metal smelter, 1960-2005. Business History, v. 50, n. 4 , p. 530-546, 2008.

LIU, X.; DAI, H.; CHENG, P. Drivers of integrated environmental innovation and impact on company competitiveness: evidence from 18 Chinese firms. Journal of Technology and Globalisation, v. 5, n. 3/4, p. 255-280, 2011.

LÓPEZ-GAMERO, M. D.; CLAVER-CORTÉS, E.; MOLINA-AZORÍN, J. F. Evaluating environmental regulation in Spain using process control and preventive techniques. European Journal of Operational Research, v. 2 n. 1, p. 497-518, 2009.

LUNDGREN, T.; MARKLUND, P.-O. Climate policy, environmental performance, and profits. Journal of Productivity Analysis, v. 44, p. 225-235, 2015.

MAÇANEIRO, M. B.; CUNHA, S. K. da; KUHL, M. R.; CUNHA, J. C. da. A regulamentação ambiental conduzindo estratégias ecoinovativas na indústria de papel e celulose. Revista de Administração Contemporânea - RAC, v. 19, n. 1, p. 65-83, jan./fev., 2015.

MANAGI, S.; OPALUCH, J. J.; DI, J.; GRIGALUNAS, T. A. Environmental Regulations and Technological Change in the Offshore Oil and Gas Industry. Land Economics, v. 81, n. 2, p. 303319, 2005.

MOHR, R. D. Technical change, external economies, and the Porter Hypothesis. Journal of Environmental Economics and Management, n. 43, p. 158-168, 2002.

MOHR, R. D.; SAHA, Shrawantee. Distribution of environmental costs and benefits, additional distortions, and the Porter hypothesis. Land Economics, v. 84, n. 4, p. 689-700, nov., 2008.

OBERNDORFER, U.; RENNINGS, K. Costs and competitiveness effects of the European Union emissions trading scheme. European Environment, v. 17, p. 1-17, 2007.

POPP, D. Uncertain R\&D and the Porter hypothesis. Contributions to Economic Analysis \& Policy, v. 4, n. 1, set., 2005.

PORTER, M. E. America’s Green Strategy. Scientific American, v. 264, n. 4, p. 96, april, 1991. 
PORTER, M. E.; van der LINDE, C. Toward a new conception of the environmentcompetitiveness relationship. Jornal of Economic Perspectives, v. 9, n. 4, p. 97-118, 1995.

RASSIER, D. G.; EARNHART, D. The effect of clean water regulation on profitability: testing the Porter hypothesis. Land Economics, v. 96, n. 2, p. 329-344, may, 2010.

RASSIER, D. G.; EARNHART, D. Short-run and long-run implications of environmental regulation on financial performance. Contemporary Economic Policy, v. 29, n. 3, p. 357-373, 2011.

RASSIER, D. G.; EARNHART, D. Effects of environmental regulation on actual and expected profitability. Ecological Economics, v. 112, p. 129-140, 2015.

RAZUMOVA, M.; IBÁÑEZ, J. L.;REY, J. Drivers of environmental innovation in Majorca hotels. Journal of Sustainable Tourism, v. 23, n. 10, p. 1529-1549, 2015.

RENNINGS, K.; RAMMER, C. The impact of regulation-driven environmental innovation on innovation sucess and firm performance. Zew -Centre for European Economic Research Discussion, n. 10-065, p. 1-34, 2010.

RUBASHKINA, Y.; GALEOTTI, M.; VERDOLINI, E.. Environmental regulation and competitiveness: empirical evidence on the Porter hypothesis from european manufacturing sectors. Energy Policy, v. 83, p. 288-300, 2015.

SADEGHZADEH, J. The impact of environmental policies on productivity and market competition. Environment and Development Economics, v. 19, n. 5, 2014.

SCHLUGA, T. R. Some micro-evidence on the "Porter hypothesis" from Austrian VOC emission standards. Growth and Change, v. 34, n. 3, p. 359-379, 2003.

SMITH, V. K.; AYERBE, C. Do Painless Environmental Policies Exist? Journal of Risk \& Uncertainty, v. 73, p. 73-94, 2000.

STELLA, B.; AGGREY, N.; ESEZA, K. Firm size and rate of growth of Ugandan manufacturing firms. Journal of Applied Economics and Business Research, v. 4, n. 3, p. 178-188, 2014.

TACHIZAWA, T. Gestão ambiental e responsabilidade social corporativa: estratégias de negócios focadas na realidade brasileira. 4. ed. São Paulo: Atlas, 2007.

TRIEBSWETTER, U.; WACKERBAUER, J. Integrated environmental product innovation and impacts on company competitiveness: a case study of the automotive industry in the region of Munich. European Environmental, v. 18, p. 30-44, 2008a.

TRIEBSWETTER, U.; WACKERBAUER, J. Integrated environmental product innovation in the region of Munich and its impact on company competitiveness. Journal of Cleaner Production, v. 16, p. 1484-1493, 2008 b. 
VLIST, A. J. van der; WITHAGEN, C.; FOLMER, H. Technical efficiency under alternative environmental regulatory regimes: the case of Dutch horticulture. Ecological Economics, v. 63, n. 1, p. 165-173, 2007.

YANG , X.; YAO, Y. Environmental compliance and firm performance: evidence from China. Oxford Bulletin of Economics and Statistics, v. 74, n. 3, p. 397-424, 2012.

YANG, C.-H.; TSENG, Y.-H.; CHEN, C.-P. Environmental regulations, induced R\&D, and productivity: evidence from Taiwan's manufacturing industries. Resource and Energy Economics, v. 34, p. 514-532, 2012.

ZIESERMER, T. A knowledge-based view of the Porter Hypothesis. Environmental Policy and Governance, v. 23, n. 3, p. 193-208, 2013. 